\title{
(6) OPEN ACCESS \\ Randomised controlled trial of a theory-based behavioural intervention to reduce formula milk intake
}

\author{
Rajalakshmi Lakshman, ${ }^{1}$ Stephen J Sharp, ${ }^{1}$ Fiona Whittle, ${ }^{1}$ Annie Schiff, ${ }^{1}$ \\ Wendy Hardeman, ${ }^{2}$ Lisa Irvine, ${ }^{3}$ Ed Wilson, ${ }^{4}$ Simon J Griffin, ${ }^{5,6}$ Ken K Ong ${ }^{1,5}$
}

\begin{abstract}
- Additional material is published online only. To view please visit the journal online (http://dx.doi.org/10.1136/ archdischild-2018-314784).

${ }^{1}$ MRC Epidemiology Unit and UKCRC Centre of Excellence in Diet and Activity Research (CEDAR), University of Cambridge, Cambridge, UK ${ }^{2}$ Health Promotion Research Group, School of Health Sciences, University of East Anglia, Norwich, UK ${ }^{3}$ Norwich Medical School, University of East Anglia, Norwich, UK

${ }^{4}$ Cambridge Centre for Health Services Research, Department of Public Health and Primary Care, University of Cambridge, Cambridge, UK

${ }^{5}$ Department of Paediatrics, University of Cambridge, Cambridge, UK

${ }^{6}$ Primary Care Research Unit, Institute of Public Health, School of Clinical Medicine, University of Cambridge, Cambridge, UK
\end{abstract}

\section{Correspondence to}

Dr Rajalakshmi Lakshman, MRC Epidemiology Unit and UKCRC Centre of Excellence in Diet and Activity Research (CEDAR), University of Cambridge, Cambridge CB2 1TN, UK; rl284@mrc-epid.cam.ac.uk

Received 12 January 2018 Revised 28 March 2018 Accepted 20 April 2018 Published Online First 14 May 2018

Check for updates

To cite: Lakshman R,
Sharp SJ, Whittle F, et al.
Arch Dis Child
2018;103:1054-1060.

\section{ABSTRACT}

Objective To assess the efficacy of a theory-based behavioural intervention to prevent rapid weight gain in formula milk-fed infants.

Design In this single (assessor) blind, randomised controlled trial, 669 healthy full-term infants receiving formula milk within 14 weeks of birth were individually randomised to intervention $(n=340)$ or attentionmatched control $(n=329)$ groups. The intervention aimed to reduce formula milk intakes, and promote responsive feeding and growth monitoring to prevent rapid weight gain $(\geq+0.67$ SD scores (SDS)). It was delivered to mothers by trained facilitators up to infant age 6 months through three face-to-face contacts, two telephone contacts and written materials.

Results Retention was $93 \%$ (622) at 6 months, 88\% (586) at 12 months and $94 \%$ attended $\geq 4 / 5$ sessions. The intervention strengthened maternal attitudes to following infant feeding recommendations, reduced reported milk intakes at ages $3(-14 \%$; intervention vs control infants), $4(-12 \%), 5(-9 \%)$ and $6(-7 \%)$ months, slowed initial infant weight gain from baseline to 6 months (mean change 0.32 vs 0.42 SDS, baselineadjusted difference (intervention vs control) -0.08 (95\% $\mathrm{Cl}-0.17$ to -0.004$)$ SDS), but had no effect on the primary outcome of weight gain to 12 months (baselineadjusted difference $-0.04(-0.17,0.10)$ SDS). By 12 months, $40.3 \%$ of infants in the intervention group and $45.9 \%$ in the control group showed rapid weight gain (OR $0.84,95 \%$ Cl 0.59 to 1.17 ).

Conclusions Despite reducing milk intakes and initial weight gain, the intervention did not alter the high prevalence of rapid weight gain to age 12 months suggesting the need for sustained intervention. Trial registration number ISRCTN20814693.

\section{INTRODUCTION}

Evidence from observational studies supports the robust and highly consistent association between rapid weight gain during infancy and later obesity ${ }^{1}$ and also with cardiovascular disease risk factors. ${ }^{2}$ In the most recent systematic review, 45/46 studies reported a positive association between infancy weight or weight gain and later childhood overweight. ${ }^{3}$ Infancy is a period of rapid growth, habit formation and developmental plasticity, ${ }^{4}$ hence it is recommended by the World Health Organization as an important time to target obesity prevention. ${ }^{5}$

Energy deposition as a percentage of total energy requirements decreases from $40 \%$ at age

\section{What is already known on this topic?}

- Rapid weight gain during infancy is consistently associated with later obesity, hence infancy could be a critical period for obesity prevention.

- Formula milk-fed infants grow faster than breastfed infants, and energy intakes of formula milk-fed infants predict weight gain and childhood body mass index.

- Although most infants are fed formula milk, no study has effectively reduced intakes among formula milk-fed infants.

\section{What this study adds?}

- This behavioural intervention reduced milk intakes and slowed initial weight gain to age 6 months, but not weight gain to 12 months.

- Infants in both groups consumed substantially higher energy than recommended and almost half showed rapid weight gain (upwards crossing $>1$ centile band) in the first year.

- Interventions to avoid rapid weight gain in infancy need to be sustained and scalable.

1 month to $1 \%-2 \%$ from 12 months until mid-adolescence. ${ }^{6}$ Therefore, weight gain during infancy is more closely related to energy intake than is weight gain in childhood or in later life. In 2004, the WHO and other international bodies reduced the estimated average energy requirements (EAR) for infants by $15 \%-20 \%$ and UK dietary reference values for energy were similarly revised in $2011 .^{6}$ However, there is wide interindividual variation among formula milk-fed infants in their energy intakes, which are positively associated with rate of infancy weight gain and childhood body mass index (BMI). ${ }^{7}$ Although the benefits of breast feeding are well recognised, only $23 \%$ of UK infants are exclusively breast fed at age 6 weeks. ${ }^{8}$ Hence, alongside breast feeding promotion, optimising the diet and growth of formula milk-fed infants may contribute to reducing the prevalence of childhood obesity.

Systematic reviews of early life interventions to prevent childhood obesity found that research in this area is recent and evolving. ${ }^{9}{ }^{10}$ The latest review in 2016 found that of 26 interventions, 7 of the 18 
behavioural interventions and two of the eight biologic interventions were effective. ${ }^{9}$

We aimed to evaluate the efficacy, mechanisms and cost of a theory-based, behavioural intervention to reduce formula milk intake and prevent excess weight gain during infancy in an explanatory, single (assessor) blind, parallel group, individually randomised controlled trial (RCT) of parents (usually mothers) and their infants.

\section{METHODS}

\section{Participants}

The full trial protocol has been published elsewhere. ${ }^{11}$ Healthy, full-term infants receiving formula milk within 14 weeks of birth were eligible to participate. Exclusion criteria were: low birth weight $(<2500 \mathrm{~g})$, preterm ( $<37$ weeks' gestation), receiving special formulas (soya-based, lactose-free, hydrolysed or antireflux formulas), major malformations and hormonal or metabolic diseases which might interfere with nutrition or growth. Participants were identified by general practitioners (42\%), research staff on a postnatal hospital ward (23\%), via a mail-out using the centralised National Health Service (NHS) integrated database 'SystmOne' (27\%), or self-referred.

\section{Intervention}

Intervention development has been previously described. ${ }^{12}$ It followed an iterative process and included systematic reviews of the literature ${ }^{13-15}$ and qualitative studies. ${ }^{16}$ The intervention included three components: a motivational component (based on social cognitive theory), ${ }^{17}$ an action planning component to help translate motivation into action (including goal setting and self-monitoring) and a coping planning component helping parents to deal with difficult situations by making 'if...then...' plans (the latter two components were based on 'implementation intentions' (online supplementary figure 1)). ${ }^{18}$ The aims of the intervention were to reduce formula milk intake (in line with 2004 WHO EAR for energy), ${ }^{6}$ and to promote responsive feeding and monitor growth to prevent excess weight gain (crossing upwards centile bands on growth charts $> \pm 0.67 \mathrm{SD}$ score (SDS)). The intervention encouraged mothers to recognise infants' satiety cues, not to force infants to finish the bottle, recognise that crying was not always due to hunger (infants may be thirsty or tired) and not to feed the infants every time they cried (try water or a dummy). It was delivered by trained facilitators (research nurses) to mothers of infants up to 6 months of age through three $30-45 \mathrm{~min}$ face-to-face sessions (at baseline and ages 4 and 6 months) and two 15-20 min telephone contacts (ages 3 and 5 months) in addition to two leaflets and stickers (with the new recommendations) to put on formula milk powder tins. We selected behaviour change techniques (BCT) with evidence of effectiveness in changing dietary behaviours, ${ }^{19}$ to target the hypothesised theory-based mediators of our intervention. We used Abraham and Michie's taxonomy ${ }^{20}$ to define the BCTs and operationalise them as intervention strategies in the intervention protocols (online supplementary table 1). The attention control group mothers received the same number of contacts during which facilitators discussed general topics including other aspects of formula milk feeding (online supplementary table 2).

\section{Outcomes}

The primary outcome was change in weight SDS from birth to 12 months. Since this was an explanatory RCT and we aimed to contribute to the sparse literature on the behavioural mechanisms of the development of childhood obesity, ${ }^{21}$ we measured a number of factors along the casual pathway (online supplementary figure 2).

Anthropometry data were collected by trained research assistants blinded to group allocation using standard operating procedures at baseline, age 6 and 12 months. Infant weight, length, BMI, and abdominal and head circumference were converted to SDS adjusted for age and sex based on the 2006 WHO Growth Standard which describes the optimal growth of healthy, breastfed children. ${ }^{22}$ Rapid/excess weight gain was defined as crossing $\geq+0.67$ SDS (one centile band). Implausible values were excluded (beyond \pm 6 SDS).

Data about the hypothesised mediators of behaviour change (maternal attitudes, self-efficacy, outcome expectancies and intentions with regard to following feeding recommendations) were collected using a validated questionnaire. ${ }^{23}$

Questionnaires at each contact (baseline, and infant's ages 3, 4, 5 and 6 months) assessed total milk intake, number of solid feeds and age at introduction of solids. ${ }^{23}$ Detailed diet data were collected at 8 months using a 4-day diet diary. The diet diary was analysed using the Diet in Nutrients Out computer package ${ }^{24}$ by the dietary assessment team at the Medical Research Council Human Nutrition Research Unit, blinded to group allocation. Health service utilisation and maternal quality of life data were collected at 6 and 12 months. ${ }^{11}$

\section{Statistical analysis}

The sample size was estimated based on a predicted difference of $0.20-0.21$ SDS in weight change from birth to 12 months assuming a 10\%-15\% reduction in milk intake between the intervention and control groups. ${ }^{7}$ Allowing for a $15 \%$ loss to follow-up, 300-350 infants in each group would provide $80 \%$ power at a two-sided 5\% significance level to detect this difference. ${ }^{11}$ Central telephone randomisation was based on a computer-generated randomisation list. All data were double-entered and cleaned by staff blinded to group allocation.

The primary efficacy outcome, change (from birth to age 12 months) in weight SDS, was analysed using linear regression with birth weight SDS and a randomised group indicator variable as covariates. The missing indicator method ${ }^{25}$ was used so that infants with missing values of a variable at birth/baseline were included in the analysis. A similar method was used for other continuous secondary outcomes. For the primary outcome, the interaction between randomised group and formula feeding status (fully/partially formula fed) was tested by including the interaction parameter in the regression model. The 'per protocol' analysis included participants completing the intervention programme based on attendance at 4/5 sessions (80\% attendance). The binary outcomes 'excess weight gain/loss' $( \pm 0.67$ SDS change from baseline) were analysed using logistic regression, with baseline weight SDS and a randomised group indicator variable as covariates. The analysis was performed using Stata V.14. ${ }^{26}$

A within-trial cost-consequences analysis from the perspective of the UK NHS comparing the Baby Milk intervention with control was conducted. Unit costs were obtained from national sources including Personal Social Services Research Unit costs and NHS reference costs. ${ }^{27} 28$

\section{RESULTS}

\section{Baseline characteristics}

Between March 2011 and June 2015, 669 infants were randomised (340 intervention, 329 control) of the 2133 assessed 


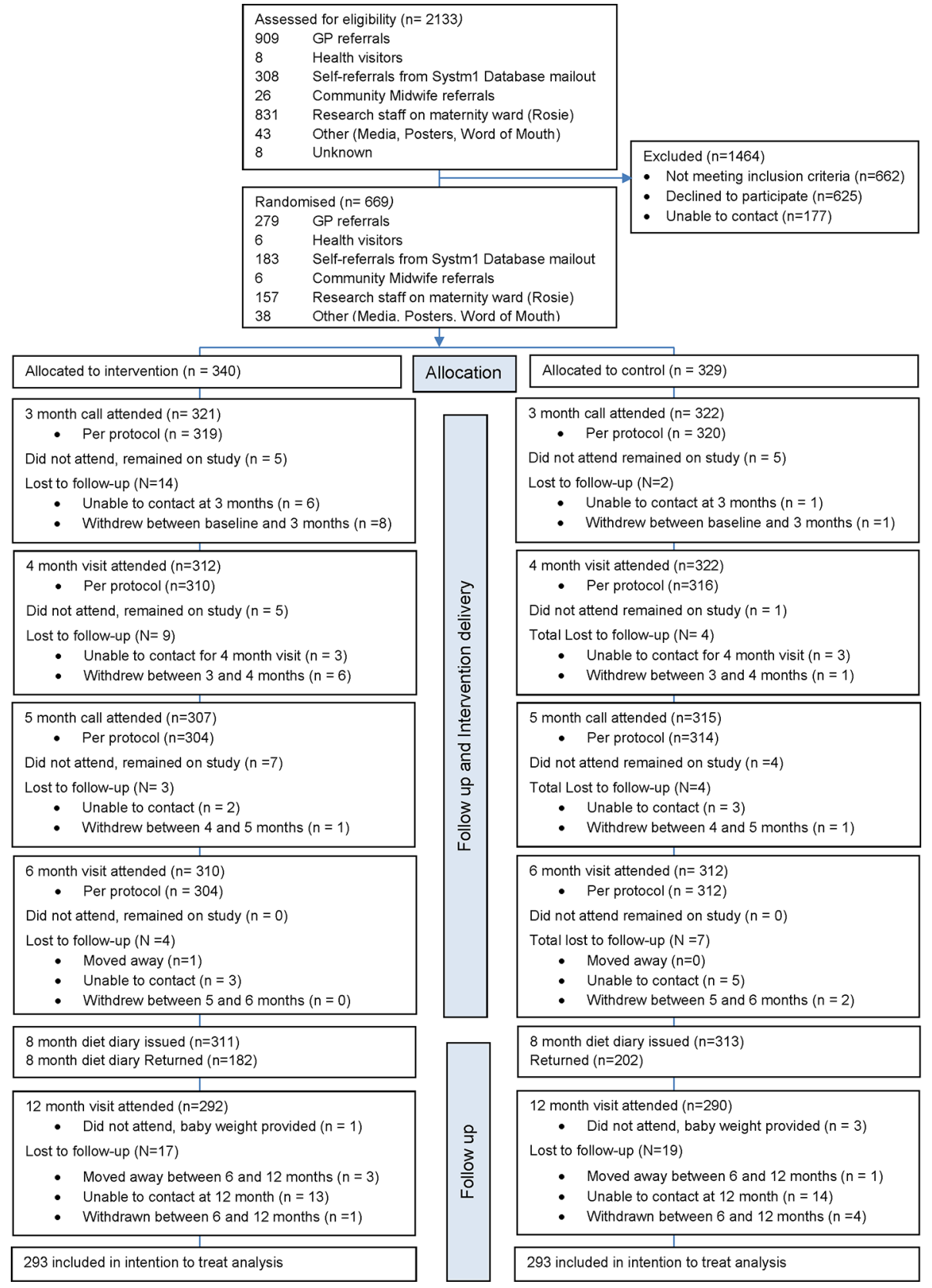

Figure 1 Trial profile. GP, general practitioner.

for eligibility (31\%). We assessed 622 infants $(93 \%$; 310 intervention, 312 control) at 6 months, and 586 infants $(88 \%$; 293 intervention, 293 control) at 12 months and this formed the intention-to-treat population (figure 1). Engagement was high in both intervention and control groups (94\%; 308 intervention, 319 control attended $\geq 4 / 5$ sessions) and 580 infants were included in the per-protocol population $(87 \% ; 288$ intervention, 292 control).

The mean (SD) age of the infants at baseline was $2.3(1.0)$ months; gestational age was 39.6 (2.0) weeks, indicating full term; birth weight was $3.4(0.5) \mathrm{kg}$ and weight at baseline $5.5(0.9) \mathrm{kg}$. Of the 669 infants, $46 \%$ were female, $94.4 \%$ fully formula milk fed at 6 months and $52.5 \%$ first born. The mean (SD) age of the mothers was 31.6 (5.8) years, BMI was $27.9(5.4) \mathrm{kg} / \mathrm{m}^{2}$ and weight gain during pregnancy was 12.9 (6.8) $\mathrm{kg}$. There were no differences in baseline characteristics among participants who completed the trial and those who were randomised (table 1).

\section{Weight gain}

Table 2 shows the between-group differences in changes in anthropometry and safety outcomes from birth/baseline to ages 6 and 12 months. The mean (SD) change in weight SDS from baseline to 6 months was $0.32(0.55)$ in the intervention group and $0.42(0.53)$ in the control group, a baseline-adjusted difference of -0.08 (95\% CI -0.17 to -0.004$)$ (figure 2). The mean (SD) change in weight SDS from birth to 12 months was $0.28(0.96)$ in the intervention group and 0.35 (1.05) in the control group, representing a difference (adjusted for birth weight SDS) of -0.04 (95\% CI -0.17 to $0.10, \mathrm{p}=0.61$ ). Results were similar in the per-protocol population. There was 
Table 1 Baseline characteristics of participants who started and completed the trial

\begin{tabular}{|c|c|c|c|c|}
\hline & \multicolumn{2}{|l|}{ Enrolled } & \multicolumn{2}{|c|}{ Completed 12-month FU } \\
\hline & $\begin{array}{l}\text { Control } \\
(n=329)\end{array}$ & $\begin{array}{l}\text { Intervention } \\
(\mathrm{n}=340)\end{array}$ & $\begin{array}{l}\text { Control } \\
(n=292)\end{array}$ & $\begin{array}{l}\text { Intervention } \\
(n=293)\end{array}$ \\
\hline \multicolumn{5}{|l|}{ Infants } \\
\hline Age (months) & $2.3(1.0)$ & $2.3(1.0)$ & $2.3(0.8)$ & $2.3(1.1)$ \\
\hline Gestational age (weeks) & $39.6(2.7)$ & $39.7(1.4)$ & $39.5(2.8)$ & $39.7(1.4)$ \\
\hline Female (\%) & $150(45.6)$ & $158(46.5)$ & $131(44.9)$ & $137(46.8)$ \\
\hline $\begin{array}{l}\text { Fully formula fed at } 6 \text { months } \\
(\%)\end{array}$ & $277(93.3)$ & 277 (95.5) & $262(93.9)$ & $264(95.7)$ \\
\hline First born (\%) & $167(53.0)$ & $174(51.9)$ & $167(53.0)$ & $174(51.9)$ \\
\hline Birth weight $(\mathrm{kg})$ & $3.41(0.5)$ & $3.47(0.5)$ & $3.41(0.5)$ & $3.45(0.5)$ \\
\hline Birth weight SDS & $0.22(1.0)$ & $0.31(0.9)$ & $0.20(1.0)$ & $0.27(0.9)$ \\
\hline Weight SDS & $-0.16(0.9)$ & $-0.06(0.9)$ & $-0.16(0.9)$ & $-0.11(0.9)$ \\
\hline BMI SDS & $-0.11(0.9)$ & $-0.03(0.9)$ & $-0.11(0.9)$ & $-0.08(0.9)$ \\
\hline Length $(\mathrm{cm})$ & $58.3(3.1)$ & $58.5(3.3)$ & $58.4(3.1)$ & $58.5(3.3)$ \\
\hline Formula milk intake (mL/day) & $898.1(219.7)$ & $895.9(217.6)$ & & \\
\hline \multicolumn{5}{|l|}{ Mothers } \\
\hline Age (years) & $31.3(5.8)$ & $31.9(5.9)$ & $31.4(5.5)$ & $32.2(5.7)$ \\
\hline $\mathrm{BMI}\left(\mathrm{kg} / \mathrm{m}^{2}\right)$ & $27.8(5.4)$ & $28.1(5.5)$ & $27.8(5.5)$ & $28.2(5.4)$ \\
\hline Pregnancy weight gain $(\mathrm{kg})$ & $12.7(6.9)$ & $13.0(6.8)$ & $12.9(6.8)$ & $13.1(6.7)$ \\
\hline $\begin{array}{l}\text { Age completed education } \\
\text { (years) }\end{array}$ & $19.5(3.6)$ & $19.6(3.5)$ & $19.6(3.2)$ & $19.6(3.4)$ \\
\hline Degree or higher & $120(38.3 \%)$ & $123(37.4 \%)$ & $110(39.1 \%)$ & $113(39.8 \%)$ \\
\hline A-level, below degree & $70(22.4 \%)$ & $72(21.9 \%)$ & $66(23.5 \%)$ & $63(22.2 \%)$ \\
\hline GCSE/vocational & $118(37.7 \%)$ & $128(38.9 \%)$ & $102(36.3 \%)$ & $103(36.3 \%)$ \\
\hline $\begin{array}{l}\text { Below GCSE or no formal } \\
\text { qualifications }\end{array}$ & $5(1.6 \%)$ & $6(1.8 \%)$ & $3(1.1 \%)$ & $5(1.8 \%)$ \\
\hline \multicolumn{5}{|l|}{ Occupation } \\
\hline $\begin{array}{l}\text { Professional, higher } \\
\text { managerial, administrative }\end{array}$ & $158(52.3 \%)$ & $142(43.3 \%)$ & $143(52.4 \%)$ & $128(44.9 \%)$ \\
\hline $\begin{array}{l}\text { Lower managerial, } \\
\text { intermediate }\end{array}$ & $93(30.8 \%)$ & $125(38.1 \%)$ & $84(30.8 \%)$ & $110(38.6 \%)$ \\
\hline $\begin{array}{l}\text { Technical, semiroutine, } \\
\text { routine }\end{array}$ & $39(12.9 \%)$ & $54(16.5 \%)$ & $35(12.8 \%)$ & $43(15.1 \%)$ \\
\hline Never employed & $12(4.0 \%)$ & $7(2.1 \%)$ & $11(4.0 \%)$ & $4(1.4 \%)$ \\
\hline White ethnicity & $295(93.1 \%)$ & $322(95.8 \%)$ & $266(93.3 \%)$ & $279(96.5 \%)$ \\
\hline Married & $184(58.2 \%)$ & $190(56.9 \%)$ & $166(58.2 \%)$ & $172(59.7 \%)$ \\
\hline Smoked during pregnancy & $38(12.0 \%)$ & $38(11.3 \%)$ & $31(10.9 \%)$ & $28(9.7 \%)$ \\
\hline $\begin{array}{l}\text { Consumed alcohol during } \\
\text { pregnancy }\end{array}$ & $64(20.2 \%)$ & $58(17.2 \%)$ & $55(19.3 \%)$ & $52(17.9 \%)$ \\
\hline
\end{tabular}

Means (SD) for continuous variables, numbers (\%) for categorical variables; SDS calculated using WHO 2006 growth charts.

BMI, body mass index; FU, follow-up; GCSE, General Certificate of Secondary Education; SDS, SD score.

no interaction between the intervention and formula feeding (fully vs partially formula fed), $\mathrm{p}=0.38$.

At 6 months, $21.8 \%$ of infants in the intervention group compared with $28.6 \%$ in the control group (OR $0.74,95 \%$ CI 0.51 to 1.07$)$ gained rapid weight $(\geq+0.67 \mathrm{SDS}$ increase from baseline). At 12 months, these proportions were $40.3 \%$ in the intervention group versus $45.9 \%$ in the control group (OR $0.84,95 \%$ CI 0.59 to 1.17 ) (online supplementary figure 3$)$.

\section{Reported milk intakes}

The average daily reported total milk intake at baseline was $897 \mathrm{~mL} /$ day, which is $5 \%$ more than the WHO EAR $(855 \mathrm{~mL} /$ day, presuming all energy is from milk). The intervention was effective in reducing milk intake (the target behaviour) at ages 3 months (mean reduction, intervention vs control: 123.5 (95\% CI 95.5 to 151.6$) \mathrm{mL} /$ day), 4 months $(115.1$ (95\% CI 87.1 to 143.0$) \mathrm{mL} /$ day), 5 months $(85.7$ (95\% CI 58.8 to 112.6$) \mathrm{mL} /$ day) and 6 months $(59.7$ (95\% CI 28.3 to
91.1) $\mathrm{mL} /$ day) (figure 3 , online supplementary table 3 ). This equated to a difference in milk intake between intervention and control groups of $-14 \%,-12 \%,-9 \%$ and $-7 \%$ at 3 , 4, 5 and 6 months, respectively. The mean (SD) age for introduction of solid feeds was 4.9 (0.84) months and over half the infants were consuming solids before the recommended age of 6 months $(2.5 \%$ at $2,5.2 \%$ at $3,24 \%$ at $4,50.4 \%$ at 5 , and $84.3 \%$ at 6 months) with no differences between groups. There was no difference between groups in the reported number of solid feeds at ages 2, 3, 4, 5 and 6 months. Average reported energy intake at age 8 months was similar in both groups $(770.1 \mathrm{kcal} /$ day vs $776.4 \mathrm{kcal} /$ day, intervention vs control) and 16\% higher than the 2004 WHO EAR (666 kcal/ day at age 7-9 months). ${ }^{6}$

\section{Maternal attitudes}

At the infant's age 6 months, the intervention increased mothers' confidence (self-efficacy) to follow the feeding recommendations in difficult situations, increased their 'expected benefits' (outcome expectancy) of following the recommendations and increased their intentions to follow the recommendations. No between-group difference was found in changes from baseline in mothers' confidence to follow the feeding recommendations without partner/family support (online supplementary figure 4).

At infant's age 6 months, the intervention increased mothers' 'worry about the baby gaining too much weight', increased 'thinking it was possible to feed the baby too much' and increased mothers' 'confidence that they could feed their baby so the baby did not gain too much weight' (online supplementary figure 5).

\section{Intervention costs}

The cost of delivering the intervention and control group protocols was estimated to be $£ 323$ and $£ 260$ per infant, respectively. The number of reported healthcare contacts was low in both groups, and there was no difference in healthcare use or costs (online supplementary tables 4 and 5).

\section{DISCUSSION}

This is the first trial of any behavioural intervention to avoid excessive energy intakes among formula milk-fed infants. The intervention reduced reported milk intakes at ages 3, 4, 5 and 6 months, and slowed initial weight gain to age 6 months. However, the effect of the intervention on weight gain was not sustained to age 12 months, the primary outcome. At age 8 months, infants in both groups consumed on average $>100 \mathrm{kcal} /$ day more total energy than their estimated average requirement.

Since almost three-quarters (73\%) of infants in the UK receive formula milk by age 6 weeks, with this proportion rising to 8 in $10(83 \%)$ by 4 months and nearly 9 in $10(88 \%)$ by 6 months, ${ }^{8}$ it is important that when mothers choose to feed their infants formula milk they are supported to feed their infants appropriately. We found that infants were reported to consume 5\% more formula milk than recommended at the start of the study (average age 2.3 months). Past attempts to reduce energy intake among formula milk-fed infants have had limited success. One trial of an educational intervention to promote responsive formula milk feeding (recognise satiety cues) did not find any difference in mean formula milk intakes at ages 4-5 months (which were $>1100 \mathrm{~mL} /$ day in both groups) and weight gain was greater in the intervention 
Table 2 Between-group differences in change in anthropometry and safety outcomes from birth/baseline to ages 6 and 12 months

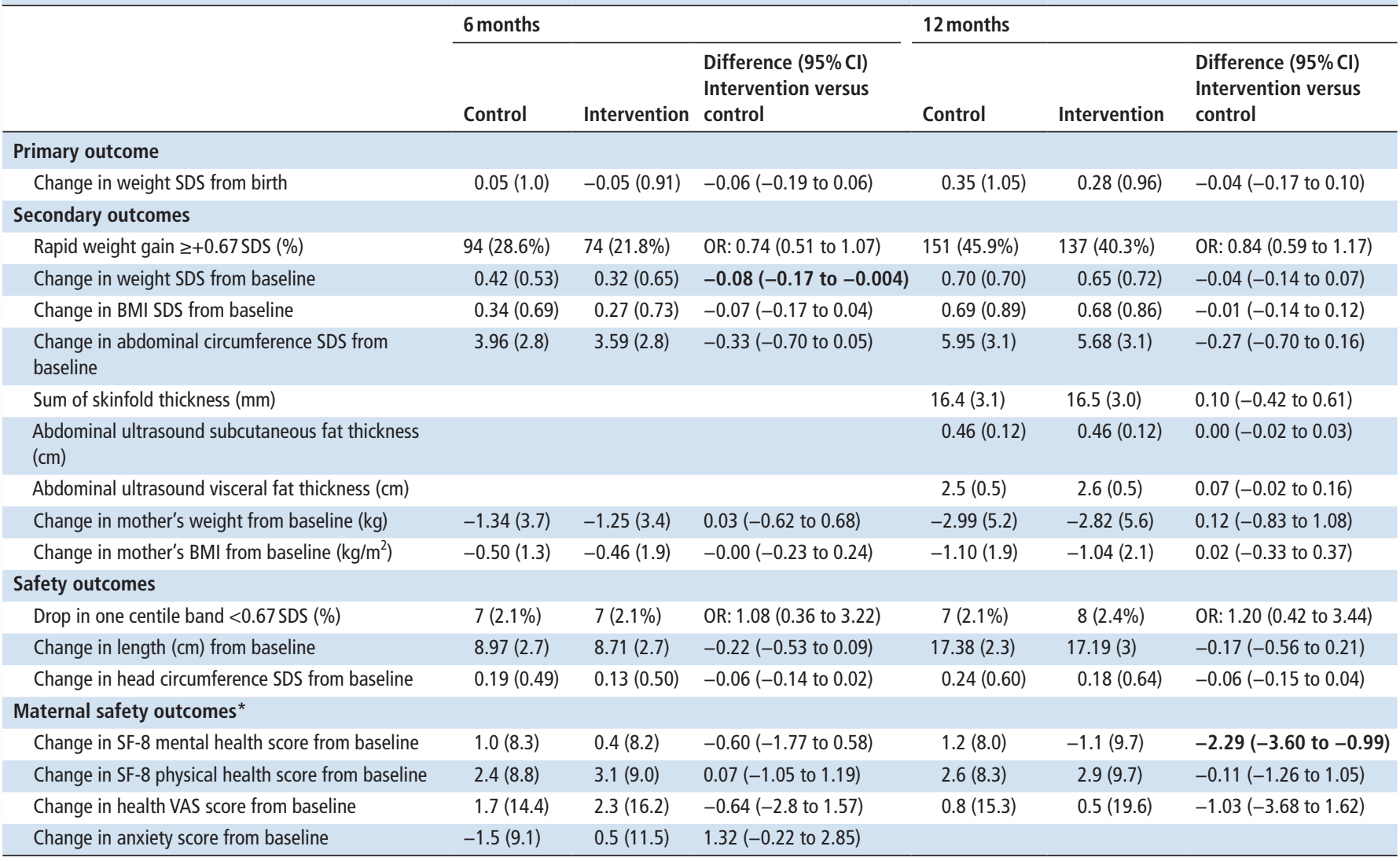

Mean (SD) for continuous variables, number (\%) for categorical variables; SDS calculated using WHO 2006 growth charts. Differences are adjusted for value of outcome at birth/ baseline if this was measured. Values displayed in bold font are statistically significant $(P<0.05)$.

*Mother's quality of life was assessed using the SF-8 and EuroQoL VAS. Maternal anxiety was measured using Spiegelberger Short State Anxiety Inventory.

BMI, body mass index; SDS, SD score; SF, Standard Form; VAS, visual analogue scale.

group than in the control group $(\mathrm{p}<0.01) .{ }^{29}$ Similarly, a trial comparing different types of bottle design found no significant differences in anthropometry at ages 2,3 and 4 months. ${ }^{30}$

Despite the high prevalence of obesity already by the age at school entry, most prevention efforts have focused on school-age children and adolescents and have had limited success. ${ }^{31}$ Studies evaluating early life interventions during

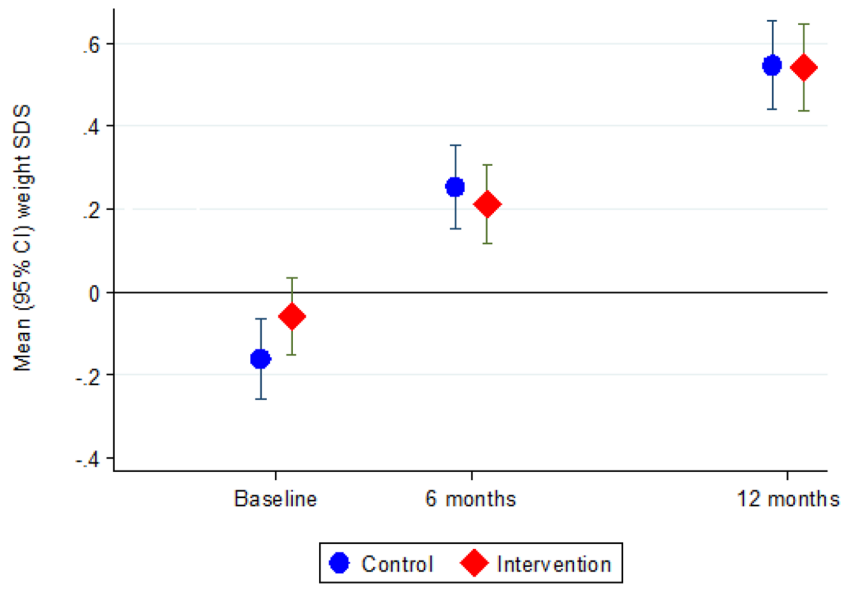

Error bars represent $95 \%$ confidence intervals

Figure 2 Weight SDS (WHO 2006 standard) in intervention and control group participants. SDS, SD score. pregnancy and the first 2 years of life are recent. ${ }^{9}{ }^{10}$ The latest systematic review (in 2016) identified 26 interventions of which nine reported a favourable effect on slower growth (7/18 behavioural, $2 / 8$ hydrolysed/lower protein formula). ${ }^{9}$ Of these, the seven effective behavioural interventions targeted maternal/family and child, sleep, diet and physical activity, and their intervention durations were invariably longer than the Baby Milk intervention (online supplementary table 6). The Baby Milk intervention adds to this body of evidence by showing that a behavioural intervention targeting formula milk intake can also have an initial favourable effect on slower weight gain when milk is the main diet. None of the behavioural interventions have shown long-term effectiveness beyond the duration of the intervention and unfortunately the Baby Milk intervention was similarly ineffective at sustaining changes beyond the intervention period. There was no difference between the two groups in reported energy intakes at 8 months and weight gain to 12 months supporting the chronic disease model for the prevention and treatment of obesity which suggests that sustained intervention may be required. ${ }^{32}$

A limitation of the trial is that participants were mainly white $(95 \%)$ although education levels were similar to the UK population $(38 \%$ had a degree or higher qualification compared to $40 \%$ of $25-40$ year olds in England and Wales). ${ }^{33}$ This could be due to the geography where recruitment took place and the motivation of mothers to take part in research with consequences for external validity and generalisability, which is not unique to our trial but a challenge for most RCTs. 


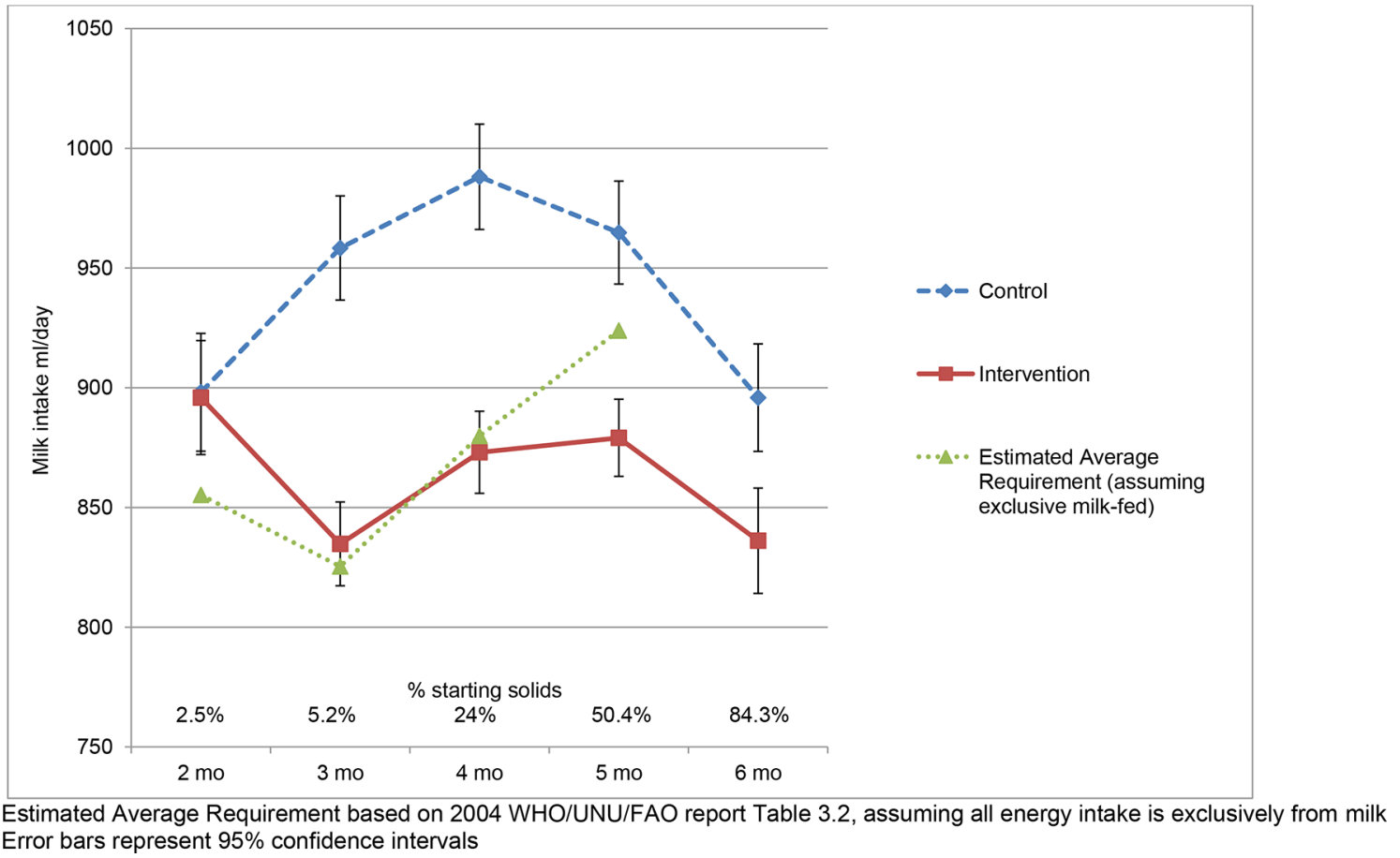

Figure 3 Milk intake (mL/day) in intervention and control group infants.

\section{CONCLUSIONS}

The high prevalence of excessive energy intakes and rapid weight gain in the Baby Milk trial highlight the importance of interventions starting in early life. Consistent with other UK data, ${ }^{34}$ we found that average reported energy intake at age 8 months (773 kcal/day) was substantially higher than the WHO estimated average requirement (666 kcal/day). Almost half (43\%) showed rapid weight gain in the first year, making this a priority area for further intervention and behaviour change. The lack of effectiveness on reported energy intakes and weight gain beyond the duration of the Baby Milk intervention suggests that future interventions need to provide sustained support that is adaptive to the changes with infant age in feeding practices and context, and may need to target other dietary behaviours, physical activity and possibly sleep. This could be feasible if supported by digital technologies, such as text messaging, mobile applications and websites. Careful development work and feasibility testing will be required to ensure that such support is based on theory and evidence, meets the needs and preferences of the target group and includes strategies to enhance initial and sustained engagement. ${ }^{35}$ Furthermore, there is a need for interventions to promote consistent and appropriate social and professional norms about a healthy pattern of growth and infant feeding. ${ }^{36}$

Acknowledgements The authors thank all the study participants and research team members, notably: Ann-Marie Wardell, Karen Forbes, Karen Poloka and Elizabeth White (intervention facilitators), Charlotte Hewitson, Sarah Arnold, Richard Powell, Chrissy-May Hall, Esther Faykeye, Ruth Watson (research assistants), Gisela Baker, Anna Thomason (study support), Emanuella De Lucia Rolfe (anthropometry specialist), Susie Boatman (data manager) and Emma Clifton (PhD student responsible for data cleaning). We acknowledge the support of the Clinical Research Network (Helen Macdonald, Janet Piggott, Brenda Deboys, Cristina Page), Cambridgeshire Community Services (Emily Li, Paula Waddingham, Elissa Harwood and David Vickers), Hertfordshire Community Services (Sally Anne Doyle-Caddick and Mary Cousins), West Anglia CLRN, general practices and hospitals that helped with participant identification. Trial Steering Committee: Andrew Clark (chair), Gail Goldberg and Gina Ambrosini (scientists), Nikki Wallis (lay member), Heather Bagley (lay member), Gavin Mattock (funder representative), James Sylvester (sponsor representative). Data Monitoring Committee: Stephen Sharp (statistician), Amanda Ogilvy Stuart (consultant neonatologist), Rachel Morris (GP). Terms of reference for
TSC and DMC have been drawn up according to the MRC/DH toolkit (http://www. cttoolkit.ac.uk/_db/_documents/Trial_MP.pdf).

Contributors RL, WH, EW, SJG and KKO are grant holders. They conceptualised the study, designed the protocol and contributed to the interpretation of data. SJS conducted the statistical analyses. LI conducted the economic analysis. FW and AS are trial managers. RL wrote the first draft of the manuscript. All authors approved the final manuscript as submitted and agree to be accountable for all aspects of the work.

Funding The Baby Milk trial is funded by the National Prevention Research Initiative (http://www.npri.org.uk Grant no. MR/J000361/1). The work was undertaken under the auspices of the Centre for Diet and Activity Research (CEDAR), a UKCRC Public Health Research Centre of Excellence which is funded by the British Heart Foundation, Cancer Research UK, Economic and Social Research Council, Medical Research Council, the National Institute for Health Research, and the Wellcome Trust. The funding partners relevant to this award are (in alphabetical order): Alzheimer's Research Trust; Alzheimer's Society; Biotechnology and Biological Sciences Research Council; British Heart Foundation; Cancer Research UK; Chief Scientist Office, Scottish Government Health Directorate; Department of Health; Diabetes UK; Economic and Social Research Council; Health and Social Care Research and Development Division of the Public Health Agency (HSC R\&D Division); Medical Research Council; The Stroke Association; Wellcome Trust; Welsh Assembly Government; and World Cancer Research Fund. RL was supported by an MRC Population Health Fellowship (Grant No G070165) and SJG, KKO and SJS are also supported by the MRC (Unit Programmes: MC_UU_12015/2 and MC_UU_12015/4).

Competing interests None declared.

Patient consent Parental/guardian consent obtained.

Ethics approval Cambridge South Research Ethics Committee (Ref: 10/H0305/9)

Provenance and peer review Not commissioned; externally peer reviewed.

Data sharing statement All raw data are available for researchers upon request.

Open access This is an open access article distributed in accordance with the terms of the Creative Commons Attribution (CC BY 4.0) license, which permits others to distribute, remix, adapt and build upon this work, for commercial use, provided the original work is properly cited. See: http://creativecommons.org/licenses/by/4.0/

(c) Article author(s) (or their employer(s) unless otherwise stated in the text of the article) 2018. All rights reserved. No commercial use is permitted unless otherwise expressly granted.

\section{REFERENCES}

1 Druet C, Stettler N, Sharp S, et al. Prediction of childhood obesity by infancy weight gain: an individual-level meta-analysis. Paediatr Perinat Epidemiol 2012;26:19-26. 
2 Leunissen RW, Kerkhof GF, Stijnen T, et al. Timing and tempo of first-year rapid growth in relation to cardiovascular and metabolic risk profile in early adulthood. JAMA 2009;301:2234-42.

3 Bja W, Locks LM, Cheng ER, et al. Risk factors for childhood obesity in the first 1,000 days: a systematic review. Am J Prev Med 2016;50.

4 Lillycrop KA, Burdge GC. Epigenetic changes in early life and future risk of obesity. Int J Obes 2011;35:72-83.

5 World Health Organization. Commission on ending childhood obesity. 2016. http:// www.who.int/end-childhood-obesity/final-report/en/

6 FAO, WHO, UNU. Human Energy Requirements. Food and Nutrition Technical, 2001.

7 Ong KK, Emmett PM, Noble S, et al. Dietary energy intake at the age of 4 months predicts postnatal weight gain and childhood body mass index. Pediatrics 2006;117:e503-e508.

8 Health and Social Care Information Centre. UK Infant Feeding Survey 2010. 2012. http://www.hscic.gov.uk/catalogue/PUB08694/Infant-Feeding-Survey-2010Consolidated-Report.pdf

9 Blake-Lamb TL, Locks LM, Perkins ME, et al. Interventions for childhood obesity in the first 1,000 days a systematic review. Am J Prev Med 2016;50:780-9.

10 Redsell SA, Edmonds B, Swift JA, et al. Systematic review of randomised controlled trials of interventions that aim to reduce the risk, either directly or indirectly, of overweight and obesity in infancy and early childhood. Matern Child Nutr 2016;12:24-38

11 Lakshman R, Whittle F, Hardeman W, et al. Effectiveness of a behavioural intervention to prevent excessive weight gain during infancy (The Baby Milk Trial): study protocol for a randomised controlled trial. Trials 2015;16:442.

12 Lakshman R, Griffin S, Hardeman W, et al. Using the Medical Research Council framework for the development and evaluation of complex interventions in a theory-based infant feeding intervention to prevent childhood obesity: the baby milk intervention and trial. J Obes 2014;2014:1-10

13 Lakshman R, Ogilvie D, Ong KK. Mothers' experiences of bottle-feeding: a systematic review of qualitative and quantitative studies. Arch Dis Child 2009;94:596-601.

14 Lakshman R, Elks CE, Ong KK. Childhood obesity. Circulation 2012;126:1770-9.

15 Wijndaele K, Lakshman R, Landsbaugh JR, et al. Determinants of early weaning and use of unmodified cow's milk in infants: a systematic review. J Am Diet Assoc 2009;109:2017-28

16 Lakshman R, Landsbaugh JR, Schiff A, et al. Developing a programme for healthy growth and nutrition during infancy: understanding user perspectives. Child Care Health Dev 2012;38.

17 Bandura A. Self-efficacy: the exercise of control. New York: Freeman, 1997.

18 Gollwitzer PM, Sheeran P. Implementation intentions and goal achievement: a meta-analysis of effects and processes. Advances in Experimental Social Psychology 2006:38:69-119.
19 Michie S, Abraham C, Whittington C, et al. Effective techniques in healthy eating and physical activity interventions: a meta-regression. Health Psychol 2009;28:690-701.

20 Abraham C, Michie S. A taxonomy of behavior change techniques used in interventions. Health Psychol 2008;27:379-87.

21 Taveras EM. Childhood obesity risk and prevention: shining a lens on the first 1000 days. Child Obes 2016;12:159-61.

22 WHO. Child Growth Standards WHO Anthro (Version 3.2.2, January 2011) and Macros. 2011 http://www.who.int/childgrowth/software/en/.

23 Lakshman RR, Landsbaugh JR, Schiff A, et al. Development of a questionnaire to assess maternal attitudes towards infant growth and milk feeding practices. Int J Behav Nutr Phys Act 2011;8:35.

24 Fitt E, Cole D, Ziauddeen N, et al. DINO (Diet In Nutrients Out) - an integrated dietary assessment system. Public Health Nutr 2015;18:234-41.

25 White IR, Thompson SG. Adjusting for partially missing baseline measurements in randomized trials. Stat Med 2005;24:993-1007.

26 StataCorp. 2015 stata statistical software: release 14. College Station, TX: StataCorp LP, 2015.

27 Department of Health. NHS schedule of reference costs 2014-15. 2015.

28 Curtis L. Unit costs of health and social care 2015. Personal Social Services Research Unit: The University of Kent, 2015.

29 Kavanagh KF, Cohen RJ, Heinig MJ, et al. Educational intervention to modify bottlefeeding behaviors among formula-feeding mothers in the WIC program: impact on infant formula intake and weight gain. J Nutr Educ Behav 2008;40:244-50.

30 Fewtrell MS, Kennedy K, Nicholl R, et al. Infant feeding bottle design, growth and behaviour: results from a randomised trial. BMC Res Notes 2012;5:150.

31 Waters E, de Silva-Sanigorski A, Burford BJ, et al. Interventions for preventing obesity in children. Cochrane Database Syst Rev 2011;165:1-191.

32 Bray GA, Kim KK, Wilding JPH. Obesity: a chronic relapsing progressive disease process. A position statement of the World Obesity Federation. Obes Rev 2017;18:715-23.

33 Office of National Statistics. Qualifications across England and Wales. 2011. http:// webarchive.nationalarchives.gov.uk/20160105190758/http://www.ons.gov.uk/ons/ rel/census/2011-census-analysis/local-area-analysis-of-qualifications-across-englandand-wales/info-highest-qualifications.html

34 Lennox A, Sommerville J, Ong K, et al. Diet and nutrition survey of infants and young children, 2011: Department of Health, 2013.

35 Yardley L, Morrison L, Bradbury K, et al. The person-based approach to intervention development: application to digital health-related behavior change interventions. J Med Internet Res 2015;17:e30.

36 Guell C, Whittle F, Ong KK, et al. Toward Understanding How Social Factors Shaped a Behavioral Intervention on Healthier Infant Formula-Feeding. Qual Health Res 2018. [Epub ahead of print 1 Mar 2018]. 